\title{
Effect of Annealing Temperature on the Electrical Resistivity of Sn-12wt\%Sb Lead Free Solder Alloy
}

\author{
A. M. Yassin \\ Physics department, Faculty of Education, Ain Shams University, \\ Cairo, Egypt
}

The effect of annealing temperatures in the range of $348 \mathrm{~K}-448 \mathrm{~K}$ on the electrical resistivity of $\mathrm{Sn}-12 w t \%$ Sb lead free solder alloy was investigated using the four-probe method. The time exponent (n) calculated from the time dependence of the isothermal electric resistivity curves has an average value of 0.02, which may be attributed to the increased number of Sb-atoms in the matrix resulting from the dissolution of the $\beta$-phase. The calculated values of the activation energy for different annealing times are ranged from 0.003 to $0.006 \mathrm{eV}$. From the electron micrographs, micro-structure investigations are also presented and discussed.

\section{Introduction:}

The electrical resistivity of metal or alloy depends largely on lattice imperfections and on the impurities it contains. Measurements of electrical resistivity are usually used to follow the precipitation and dissolution processes resulting from the thermal treatments of alloys [1-4]. Ari et al. investigated the thermo-electrical properties of $\mathrm{Sn}-\mathrm{Zn}$ alloys for different compositions. They found that the resistivity of samples increased linearly with temperature and the temperature coefficient of electrical resistivity was independent on the composition of $\mathrm{Sn}$ and $\mathrm{Zn}$ [5]. Mhiaoui et al. have measured the resistivity of lead free solders and they found that it is smaller than that of lead-tin alloys [6].

The dissolution of the $\gamma$-phase in Al-Mg alloy has been studied and it was found that the rate of $\gamma$-phase dissolution followed an Avrami-type equation with the time exponent $\mathrm{n}=0.55-0.70$ [7]. Shinoda et al. [8] studied the dissolution of the $\varepsilon$-phase precipitation formed in a $\mathrm{Cu}-6 \mathrm{wt} \% \mathrm{Sn}$ alloy. They measured the electrical resistivity in the temperature range $400-550{ }^{\circ} \mathrm{C}$ and found that the electrical resistivity decreased by increasing the pre-cold working [8]. 
El-Daly et al. [9] measured the electrical resistivity of $\mathrm{Pb}-65.5 \mathrm{Sn}-3.4 \mathrm{Zn}$ eutectic and $\mathrm{Pb}-65.0 \mathrm{Sn}-1.0 \mathrm{Zn}$ ternary alloys at various ageing temperatures for various ageing times. They found that the $\mathrm{Pb}-\mathrm{Sn}-\mathrm{Zn}$ ternary alloys show reduction in the electrical resistivity compared with the $\mathrm{Pb}-\mathrm{Sn}$ binary eutectic composition. This is a result of the nature of the internal stresses induced by the $\mathrm{Zn}$ particles and the multiplicity of types of inter-phase boundary that causing enhancement in the driving force for the coarsening process. Hirano and Nagai [4] studied the dissolution of the $\gamma$-phase in the Al-Ag alloys and they found that the rate of dissolution of the $\gamma$-phase follows an equation of the type:

$$
\begin{aligned}
& \mathrm{x}=\left(\rho_{\mathrm{t}}-\rho_{\mathrm{o}}\right) /\left(\rho_{\infty}-\rho_{\mathrm{o}}\right)=\mathrm{kt}^{\mathrm{n}} \\
& \mathrm{x}=\Delta \rho / \Delta \rho_{\infty}=\mathrm{kt}^{\mathrm{n}} \\
& \Delta \rho=\Delta \rho_{\infty} \mathrm{kt}^{\mathrm{n}} \\
& \Delta \rho=\mathrm{k}^{\prime} \mathrm{t}^{\mathrm{n}}
\end{aligned}
$$

where: $x$ is the fraction of electrical receptivity change during dissolution.

$\rho_{0}, \rho_{t}$ and $\rho_{\infty}$ are, respectively, the electrical resistivity of as-received specimen at room temperature $\left(25^{\circ} \mathrm{C}\right)$, the resistivity at time $\mathrm{t}$ and the final resistivity after an infinite time of annealing.

$\mathrm{k}$ is the rate constant of the precipitate.

$\mathrm{n}$ is the time exponent.

$\mathrm{k}^{\prime}$ is another constant proportional to the rate constant $\mathrm{k}$.

The aim of the present work is to investigate the effect of annealing temperatures on the electrical resistivity of $\mathrm{Sn}-12 \mathrm{wt} \% \mathrm{Sb}$ lead free solder alloy ( $\beta$-phase of the intermetalic compound $\mathrm{SnSb}$ ).

\section{Experimental work:}

\subsection{Preparation of the alloy:}

This study has been carried out on a Sn- $12 \mathrm{wt} \% \mathrm{Sb}$ alloy, which was prepared from high purity (99.99\%) component materials. All components were ultrasonically cleaned, which removed surface contamination and reduced the possibility of introducing impurities into the alloys. The weighed materials were melted under vacuum and casting was done in a graphite mould. The ingots (rods of $12 \mathrm{~mm}$ in diameter) were swaged to form wires of diameter $0.6 \mathrm{~mm}$. 


\subsection{Heat Treatment:}

The annealing process was carried out isothermally in an automatic furnace provided with a thermo regulator. The temperature inside the furnace was maintained constant within $\pm 1^{\circ} \mathrm{C}$. Alloy specimens of length $9 \mathrm{~cm}$ were annealed at different temperatures $348,373,398,423$ and $448 \mathrm{~K}$ for a period from 10 to $90 \mathrm{~min}$ at each temperature in steps of $5 \mathrm{~min}$. Then, they rapidly quenched in water at $298 \mathrm{~K}$ (room temperature) to obtain specimens of different preliminary microstructures.

\subsection{Electrical Resistivity Measurement:}

The electrical resistivity of the annealed samples were isothermally measured at room temperature using the four-probe method, where the four electrical contacts were mad by using silver paints in conjunction with copper wire. Each contact is perimetrically around the samples strip. The outer two contacts are for passing DC current and the inner two contacts are for measuring the voltage. A Keithley 2002 multimeter was used.

\subsection{Microstructurse Measurements:}

The specimens were polished conventionally using decreasing grades of silicon carbide paper. Tin-antimony alloys are more difficult to etch than other tin-base alloys. An etching solution of $2 \%$ nital was used for about $2 \mathrm{sec}$, which darkens the tin-rich matrix, leaving intermetallics ( $\beta$-phase) unattached. For scanning electron metallography, samples had to be lightly etched and it was necessary to examine them fresh.

\section{Results:}

The isothermal electrical resistivity curves for annealed $\mathrm{Sn}-12 \mathrm{wt} \% \mathrm{Sb}$ alloy are shown in Fig. (1). The change in electrical resistivity was calculated at different annealing times to follow the various precipitation processes during the annealing temperature. The resistivity change increases with increasing the annealing temperature.

The time exponent $\mathrm{n}$ from Eqn. (2) was calculated by plotting $\ln (\Delta \rho)$ against $\ln (\mathrm{t})$ as shown in Fig. (2) for samples isothermally annealed at different temperatures. The activation energy was calculated from the slope of the linear relation between $\ln \left(\rho_{t} / \rho_{o}\right)$ and $(1000 / T)$ for different annealing times. It represents the formation process of precipitates. The calculated values of the activation energies ranged from 0.003 to $0.006 \mathrm{eV}$.

Electron microscope investigation is carried out for the microstructure of Sn-12wt $\% \mathrm{Sb}$ alloy. Figures ( $4 \mathrm{a}$ and $\mathrm{b}$ ) show samples annealed at $373 \mathrm{~K}$ and $448 \mathrm{~K}$ for $30 \mathrm{~min}$, respectively. 


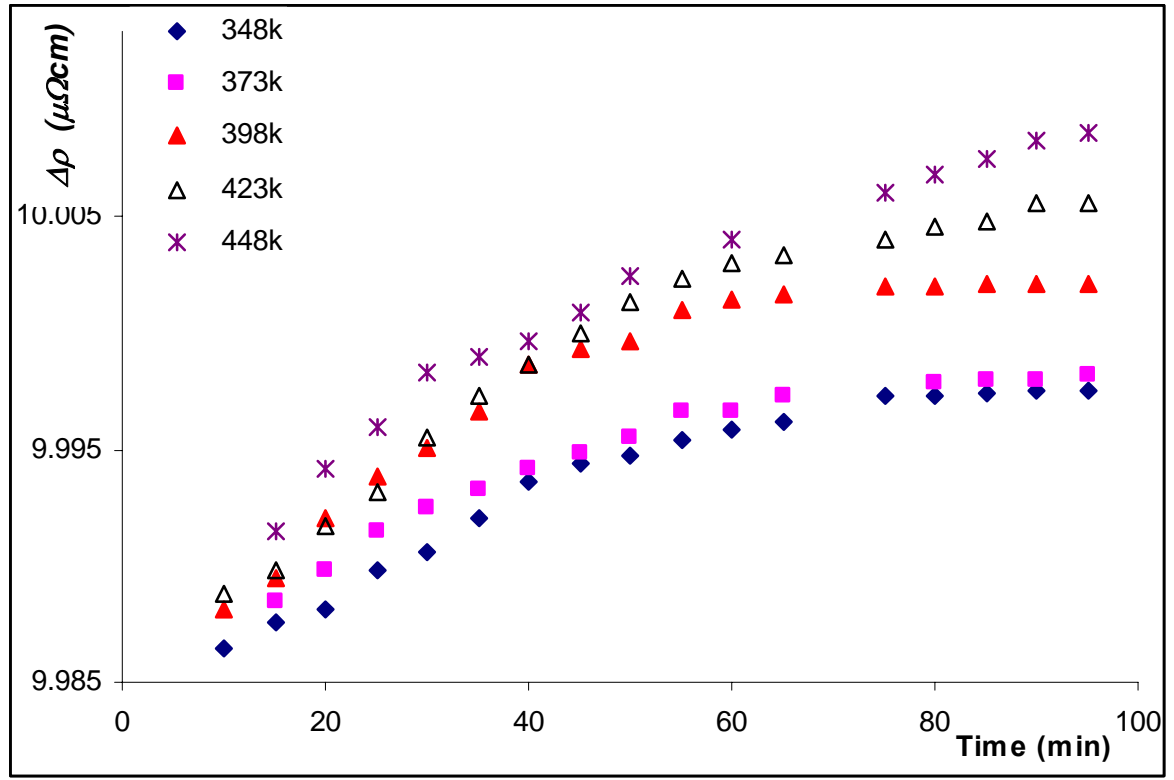

Fig. (1): The relation between the electrical resistivity change $(\Delta \rho)$ and annealing time for different annealing temperature.

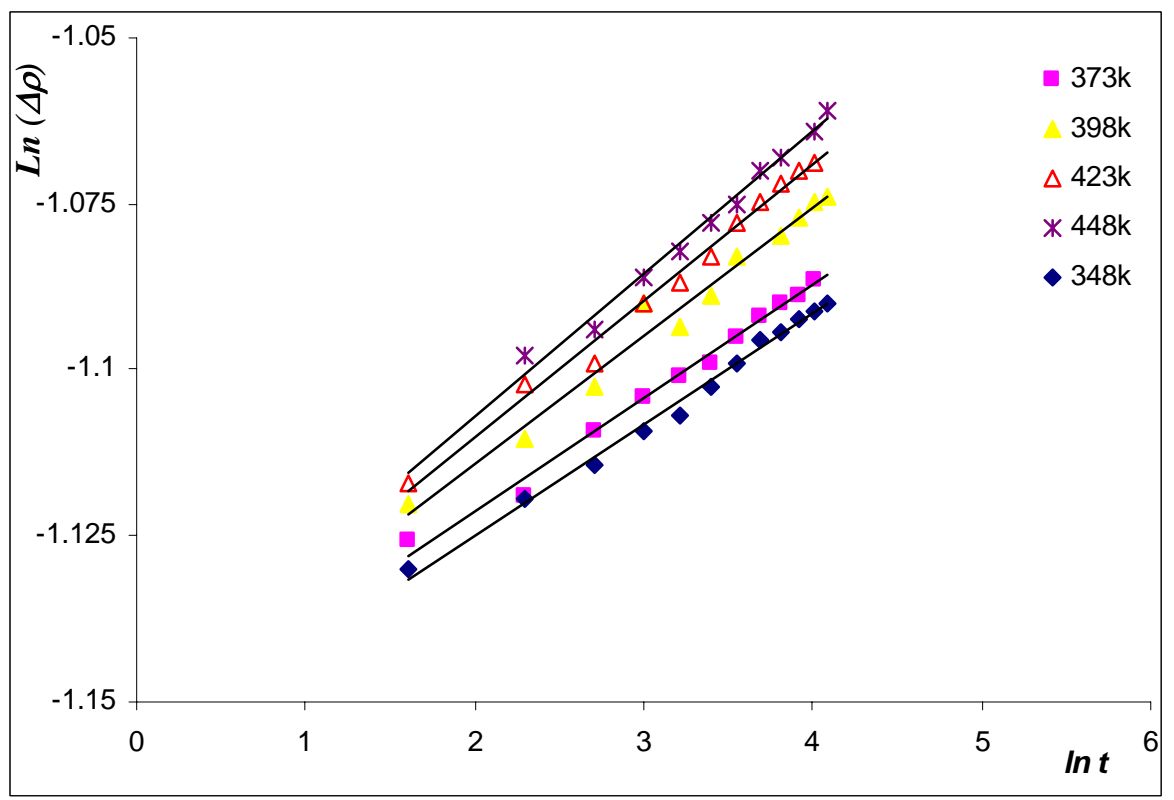

Fig. (2): The relation between $\ln (\Delta \rho)$ and $\ln (\mathrm{t})$ for different annealing temperature from $(348-448 k)$. 


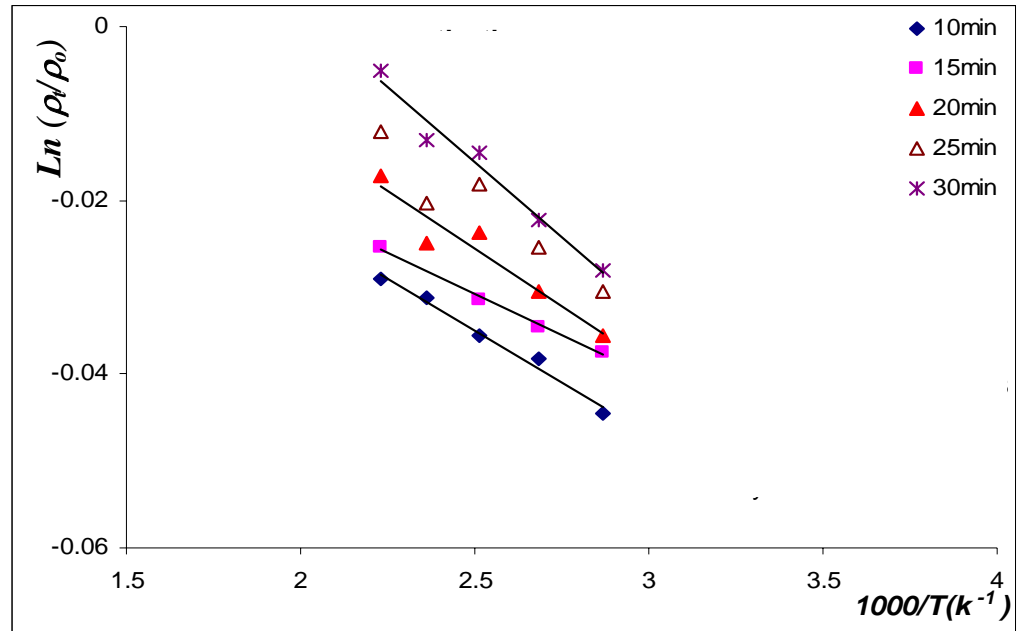

Fig. (3): The relation between $\ln \left(\rho_{t} / \rho_{o}\right)$ and the reciprocal of the annealing temperature $(1000 / T)$ for different annealing times.

(a)

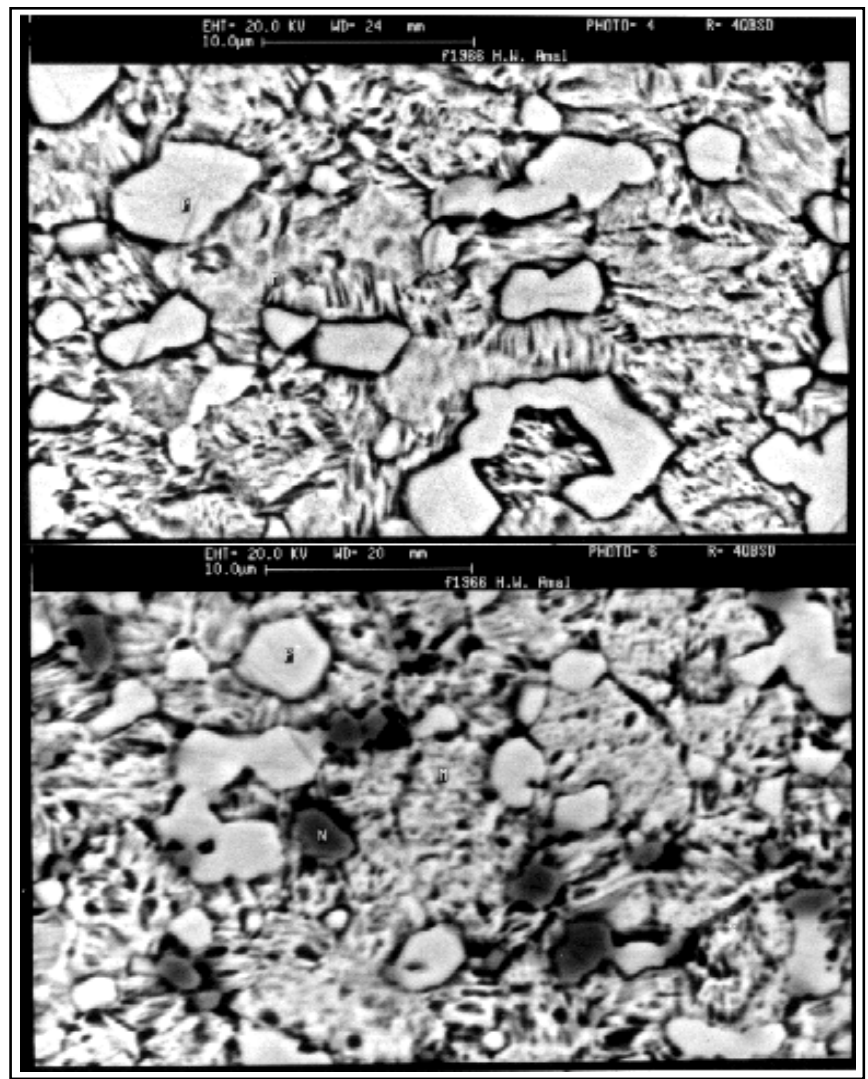

Fig. (4): The electron micrograph of annealing $\mathrm{Sn}-12 \mathrm{wt} \% \mathrm{Sb}$ alloy for $30 \mathrm{~min}$ (a) at 348 $\mathrm{K}$ and (b) at $448 \mathrm{~K}$. 


\section{Discussion:}

The dependence of the electrical resistivity change of the Sn- $12 \mathrm{wt} \% \mathrm{Sb}$ alloy on the annealing temperatures is shown in Fig. (1). The electrical resistivity at the beginning increased gradually by increasing the annealing time and then seems to be constant. It has been assumed that there is an explicit solid solution strengthening effect caused by antimony dissolved in tin. Furthermore, the $\mathrm{SnSb}$ intermetallic phase is only marginally different to an ordered solid solution [10] and its relatively slight effect in strengthening tin has been noted compared, for example, with $\mathrm{Cu}_{6} \mathrm{Sn}_{5}$ [11]. Since the effect of annealing is to reduce the volume fraction of $\mathrm{SnSb}$, increase the amount of antimony dissolved in the matrix and to increase the mean grain size, it could be argued that the entire effect of annealing is due to changes in grain size. The fine particles of $\beta$-phase $(\mathrm{SnSb})$ can be treated as isolated scattering centres for conduction electrons. Accordingly, the scattering of conduction electrons increases spontaneously with the increasing of the tiny $\beta$-phase. The resistivity increases due to the decrease in the size of the $\beta$-phase ( $\mathrm{SnSb}$ ) using vacancy hopping.

As the annealing temperature increases, the dissolution of $\beta$-phase might be associated with an intensive diffusion current of vacancies. This causes the electrical resistivity change to increase with increasing of the concentration of vacancies which rises gradually with increasing both annealing temperature and annealing time. The time exponent $\mathrm{n}$ has been estimated from slopes of parallel straight lines in Fig. (2) and it has an average value of 0.02. This may be attributed to the increased number of Sb-atoms in the matrix resulting from the dissolution of the $\beta$-phase at high rate. The dissolved atoms can be treated as isolated scattering centres for the conduction electrons.

The activation energy value of dissolution processes of the annealing, as estimated from Fig.(3), was found to be in the range of $0.003-0.006 \mathrm{eV}$, which may be due to the binding energy between vacancy and an antimony solute atom [7].

To support the electrical resistivity measurements, two of the annealing temperatures ( 348 and $448 \mathrm{~K}$ ) were chosen to study the effect of annealing temperature on the proportion of $\mathrm{SnSb}$ in the microstructure. Each anneal was carried out for $30 \mathrm{~min}$ followed by quenching into water at a temperature of $298 \mathrm{~K}$, which causes $\mathrm{SnSb}$ to dissolve allowing the mean free distance in the $\alpha$ phase to increase, whereas both the $\mathrm{SnSb}$ islet size and volume fraction decrease. Figures ( $4 a$ and $b)$ illustrated the general effects of annealing temperature on the $\mathrm{Sn}-12 \% \mathrm{Sb}$ alloy. The effect of annealing is, therefore, to tend the volume fraction of $\mathrm{SnSb} \beta$-phase towards the equilibrium amount. These results in a reduction of the volume fraction and an accompanying 
increase of the amount of $\mathrm{Sb}$ dissolved in the tin-rich $\alpha$-phase. Alternatively, $\mathrm{SnSb}$ may have re-precipitated as sub-microscopic particles, perhaps as the semi-coherent needles reported by Kaczorowski and Matyja [10].

\section{Conclusion:}

The time exponent (n) calculated from the time dependence of the isothermal electric resistivity curves has an average value of 0.02 . This may be attributed to the increased number of Sb-atoms in the matrix resulting from the dissolution of the $\beta$-phase at high rate. The calculated values of the activation energy for different annealing times are ranged from 0.003 to $0.006 \mathrm{eV}$. It represents the formation process of precipitates. From the electron micrographs, the effect of annealing is to tend the volume fraction of $\mathrm{SnSb} \beta$-phase towards the equilibrium amount.

\section{References:}

1. H. El-Sayed, and I. Kovaces, phys. stat. sol. (a) 27, k35 (1975).

2. N. Luggi, J. P. Simon and P. Guyot, Acta Met. 28, 1115 (19800.

3. M. A. Kenawy, M. R. Nagy, M. S. Sakr and M. T. Mostafa, phys. stat. sol., (a) 97, k73 (1986).

4. Ken-ichi Hirano, and Katsu Nagai, Metallurgical Abstracts on Light Metals and Alloys, VIII, published by the Light Metals Educational Foundation, Osake, Japan 1974.

5. M. Ari, B. Saatci, M. Payveren and S. Durmus, Materials Characterization, 59 (6), 757 (2008).

6. S. Mhiaoui, F. Sar and J. G. Gasser, Journal of Non-Crystalline Solids, 353 (32-40), 3628 (2007).

7. M. A. Kenawy, M. S. Sakr, H. A. Zayed, E. M. Sakr, and N. O. Mourad, Egypt. J. Solids, 14 (1), 25 (1991).

8. R. Shinoda, A. Fawzy, G. Graiss and G. Saad, Solid State Communications, 84 (3), 335 (1992).

9. A. El-Daly, A. M. Abdel-Daiem and M. Yousf, Materials Chemistry and Physics, 78 (1-3), 73 (2003).

10. M. Kaczorowski and H. Matyja, J. Materials Science, 14, 2887 (1979).

11. E. S. Hedges, "Tin and its Alloys", Edward Arnold, London (1960). 